\title{
空时社交关系池化行人轨迹预测模型
}

\author{
毛琳，巩㸝飞*，杨大伟，张汝波 \\ (大连民族大学机电工程学院 大连 116600) \\ (chengshux@foxmail.com)
}

\begin{abstract}
摘 要: 针对社会对抗网络行人轨迹生成模型(SGAN)对行人长时社交关系考虑不足的问题，提出一种空时社交关系 池化行人轨迹预测模型. 通过空时社交汇集机制学习行人观测序列的全部社交，获得空时映射的社交汇集向量，再 利用关系池化的方法, 将空时社交汇集向量池化为“引力-斥力”关系的社交向量, 作为 RNN解码器隐藏态输人的一部 分, 使模型既能保持行人短时社交敏感性，又能增强长时社交关系的记忆，提高模型对行人复杂社交下的预测精度. 为了验证提出模型的可靠性, 使用公开标准数据集 ETH 和 UCY 来测试性能, 实验表明模型相比于 SGAN, 平均偏移 精度误差提升 $20 \%$ ，最终偏移精度误差提升 $13.9 \%$.
\end{abstract}

关键词: 轨迹预测; 空时社交汇集; 关系池化

中图法分类号: TP391.4 DOI: 10.3724/SP.J.1089.2020.18236

\section{Space-Time Social Relationship Pooling Pedestrian Trajectory Prediction Model}

\author{
Mao Lin, Gong Xinfei*, Yang Dawei, and Zhang Rubo \\ (College of Mechanical and Electronic Engineering, Dalian Minzu University, Dalian 116600)
}

\begin{abstract}
Socially acceptable trajectories with generative adversarial networks (SGAN) does not consider the long-term social relationships of pedestrians, a spatial-temporal social relationship pooling pedestrian trajectory prediction model is proposed. The spatial-temporal social collecting mechanism is used to learn all the social interactions of the pedestrian observation sequence and obtain the social collecting vector of spatial-temporal mapping. And then the social relationship pooling method is applied to pooling the spatial-temporal social collecting vectors into social vectors of "gravity-repulsion" relationships, as a part of the hidden state input of the RNN decoder. The model can not only maintain the short-term social sensitivity of pedestrians, but also enhance the memory of long-term social relationships, and improves the model's prediction accuracy for pedestrians with complex social relationships. In order to verify the reliability of the proposed model, the performance was tested on the public standard data sets ETH and UCY. Experiments show that the average offset accuracy error of our model is increased by $20 \%$ compared with the SGAN model, and the final offset accuracy error is increased by $13.9 \%$.
\end{abstract}

Key words: trajectory prediction; spatial-temporal social collect; relationship pooling

随着无人驾驶 ${ }^{[1]}$ 、智能交通 ${ }^{[2]}$ 和安全监控等应 用技术的发展, 行人轨迹预测逐渐成为一个研究
热点. 尤其在一些行人聚集的区域，由于多目标行 人具有极其复杂的运动方式, 其轨迹往往难以估

收稿日期：2020-02-15; 修回日期：2020-07-13．基金项目：国家自然科学基金(61673084); 辽宁省自然科学基金(20170540192, 20180550866). 毛琳(1977一), 女, 博士, 副教授, 硕士生导师, 主要研究方向为目标跟踪与多传感器信息融合; 巩欣飞(1994一), 男, 硕士研究生, 论文通讯作者, 主要研究方向为行人轨迹预测; 杨大伟(1978一), 男, 博士, 副教授, 硕士生导师, 主要研究方向为计算 机视觉处理技术; 张汝波(1962-), 男, 博士, 教授, 硕士生导师, 主要研究方向为智能机器人技术及智能信息处理技术. 
计. 行人轨迹预测算法主要是通过学习行人已有 轨迹的运动规律, 准确预测出后续轨迹, 提供多目 标在空间中的连续位置信息, 为无人车等应用提 供预处理环境感知信息.

近几年, 随着深度学习的兴起, 采用循环神经 网络(recurrent neural networks, RNN)的行人轨迹 预测算法有了长足的发展，极大提升了预测精度. 早期的行人轨迹预测算法采用基于物理运动规律 的预测方法 ${ }^{[3]}$, 能较好地预测匀速直线运动条件下 行人轨迹, 但对复杂行人轨迹预测精度较低, 也未 考虑行人互动对轨迹的影响. 经典的轨迹预测算 法有 Helbing 等 ${ }^{[4]}$ 提出的社会力模型，该模型以牛 顿力学法则为基础, 将每个行人视为一个受到多 种力的质点, 推动每个人自身运动的力定义为自 驱力, 行人的互相影响量化为吸引力和排斥力, 每 种施加的力对行人产生不同矢量的速度和加速度, 综合计算所得结果即为行人的预测轨迹. 该模型 对多行人的社交化影响解决较差，适用于解决个 体互动较少的情况.

随着深度学习的发展，长短时记忆网络 ${ }^{[5]}$ (long short-term memory, LSTM)被广泛应用于与时序有 关的问题, 而行人轨迹本质上是一组时序数据, 通 过引人 LSTM, 学习行人运动的时序特征, 预测出 未来时刻的轨迹, 但未解决行人社交化对轨迹的 影响. 针对这个问题, Alahi 等 ${ }^{[6]}$ 提出拥挤空间中的 人体轨迹预测模型(social LSTM, S-LSTM), 每个行 人的轨迹预测都由一个独立的 LSTM 负责来学习 对应的观测时序，同时利用社交网格池化层对行 人与其他个体的空间信息进行汇集，形成一个可 以包含所有行人空间信息的社交汇集向量. 在每 个 LSTM 由上一时刻到下一时刻传递隐藏态时, 将社交汇集向量映射到隐藏态的同一空间，作为 下一时刻的隐藏态输人, 输出社交化影响下的预 测轨迹. 但该模型中行人的社交池化需要大量的 手工计算, 对复杂情况下行人社交泛化能力不足. Gupta 等 ${ }^{[7]}$ 提出社会生成对抗网络(social generative adversarial networks, SGAN)行人轨迹生成模型, 生成对抗网络 ${ }^{[8]}$ 可以避免使用单一的损失函数来 衡量输出轨迹, 通过观测轨迹尽可能学习行人运 动的空间分布特点. 生成对抗网络由生成器和判 别器组成, 生成器由 RNN 编码-解码器 ${ }^{[9]}$ 和社交池 化模块组成, 负责生成行人的多种轨迹, 判别器负 责对生成轨迹进行判定. SGAN 采用一种新的社交 化向量汇集机制，该方法通过行人的空间信息线 性变换后, 与 RNN 编码-解码器隐藏态统一编码,
避免手工计算方法的缓慢和信息不全的问题．该 模型存在的问题是过于关注短时社交，导致预测 轨迹极大地被短时社交影响。

行人轨迹预测在各种应用场景中面临着多种 挑战. 针对现有方法大多忽略行人交互的时间作 用问题, Huang 等 ${ }^{[10]}$ 提出时空交互行人轨迹模型 STGAT，通过时空图注意机制来学习行人交互的 时间相关性, 提高模型对多行人互动下轨迹的预 测精度. 针对行人社交和行人与场景空间物理互 动的问题, Kosaraju 等 ${ }^{[11]}$ 提出一种基于图注意网络 和 Bicycle-GAN 的多模态行人轨迹预测方法Social-BiGAT, 通过图生成对抗网络学习场景中行 人的社交, 利用图注意网络对行人与场景的空间物 理互动编码, 输人解码器生成多模态的行人轨迹.

\section{1 行人轨迹社交化问题}

行人轨迹预测算法是利用载有行人的视频, 将目标在三维真实世界中固定时间间隔下的观测 坐标输人模型, 预测出行人在未来连续时间内的 轨迹. 行人轨迹预测算法可分为人-空间作用和人人相互作用 2 类. 人-空间作用类旨在研究行人在特 定空间下的运动方式 ${ }^{[12]}$, 如行人在充满障碍物的空 间中的行进轨迹路线; 人-人相互作用类研究的是 多个行人在同一空间社交影响下的轨迹预测 ${ }^{[13]}$.

本文研究的是人-人相互作用类的多行人轨迹 预测. 难点有 3 个方面：(1) 由于空间中的每个行 人轨迹都可以视为一个单独的时序, 预测应满足 行人在三维空间中的物理运动规律, 但由于多个 行人处于同一空间，行人之间的相互影响变得不 可避免，如何保证算法的预测结果既能满足个体 行人的物理规范, 又能考虑到行人的彼此影响. (2) 在多行人的轨迹预测中, 个体存在着社交需求的 特殊性. 如一对朋友相伴而行或朋友迎面放慢脚 步打招呼等，都会使行人轨迹存在一种异于其他 个体的社交化影响，并且这种社交化关系成为影 响行进轨迹的最重要因素. (3) 满足多行人的预测 轨迹存在多个解, 如何找出其中的最优解是关键.

SGAN 模型作为目前最优秀的轨迹预测模型 之一, 其存在的问题是只利用观测的最后轨迹点 来分析行人社交, 没有利用观测序列的全部社交 关系，导致模型过于关注短时社交. 从现实角度考 虑，一个稳定的社交关系往往表现为一种长期接 近的空间距离, 而不是短时接近, 如一对朋友的轨 迹在空间中会表现为长期相伴. 以公开数据集某 
一行人序列的实验为例, SGAN 的轨迹预测和真实 轨迹如图 1a 所示, 行人 1 和行人 2 的真实轨迹交 叉穿行, 先接近后偏离. 从观测序列的长期轨迹点 来看, 不具备稳定的社交可能化, 但由于 SGAN 池 化网络只学习观测序列最后一个轨迹点的社交关 系，使得模型认为短期接近是一个稳定的社交关 系, 从而预测两者的轨迹为连续接近, 与真实轨迹 不符.

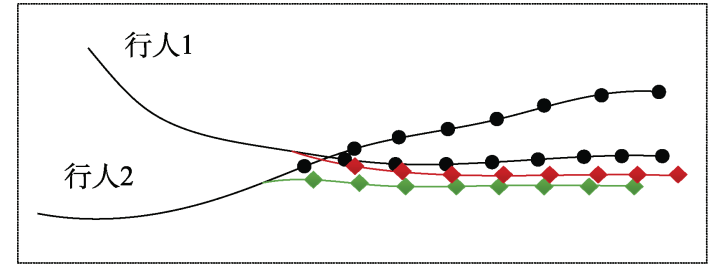

a. 同向而行

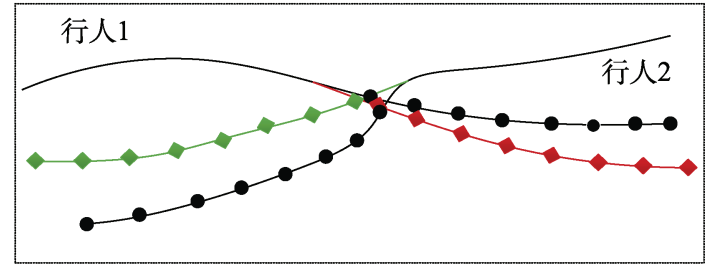

b. 相向而行

一观测轨迹; - - 真实轨迹; $\diamond \prec$ 预测轨迹

图 1 行人的真实轨迹与预测轨迹

SGAN 模型的另一个问题是对行人社交关系 考虑不足, 如图 $1 \mathrm{~b}$ 所示, 行人 1 和行人 2 是一对相 向而行的陌生行人，行人 1 和行人 2 没有长期亲近 社交化的可能，且由于两者行进方向相反，轨迹路 线接近，使得两人存在“相撞”的社交可能. 在行人 2 的真实轨迹, 做出了避让的决策, 但在 SGAN 的 预测中，并没有考虑到这一社交关系，使得行人 2 的预测轨迹失效.

针对 SGAN 对行人长时社交关系考虑不足的 问题，本文提出一种空时社交关系池化行人轨迹
预测模型 (space-time social relationship pooling pedestrian trajectory prediction model, SOPM), 通 过空时社交汇集机制将行人的全部历史社交信息 传递给社交关系池化模块, 转换成空时社交池化 向量，提高模型行人长时社交记忆.

\section{SOPM}

\section{1 问题描述}

定义 1. 已知所有行人的观测序列为 $X=$ $\left(X_{1}, \cdots X_{i}, \cdots, X_{n}\right)$, 其中, $i$ 为人群中的行人编号, $n$ 为空间中所有行人的个数, $n \in \mathbb{Z} . X_{i}=$ $\left[X_{i}^{1}, \cdots, X_{i}^{t}, \cdots, X_{i}^{t_{\mathrm{obs}}}\right]$, 其中, $t_{\mathrm{obs}}$ 为观测序列的长度, $X_{i}^{t}=\left(x_{i}(t), y_{i}(t)\right)$. 行人轨迹算法的任务是在已有 的观测序列的基础上，通过分析计算得出未来时 刻的预测序列 $Y=\left(Y_{1}, \cdots, Y_{i}, \cdots, Y_{n}\right)$, 其中, $Y_{i}=$ $\left[Y_{i}^{t_{\text {obs }}+1}, \cdots, Y_{i}^{t}, \cdots, Y_{i}^{t_{\text {obs }}+t_{\text {pred }}}\right], t_{\text {pred }}$ 为预测序列的长度, $Y_{i}^{t}=\left(x_{i}(t), y_{i}(t)\right)$. 为了区别算法输出的预测序列 和行人的真实轨迹, 设输出的预测序列为 $\hat{Y}$, 行人 的真实轨迹为 $Y$.

\section{2 模型网络结构}

空时社交关系池化行人轨迹预测模型如图 2 所示, 生成对抗网络中的生成器来拟合行人的未 来轨迹, 判别器负责对预测轨迹判定. 生成器由 RNN 编码-解码器和空时社交关系池化网络组成, 编码器通过学习行人的观测序列获得隐藏态, 空 时社交关系池化网络汇集行人社交关系信息后池 化, 再由隐藏态和社交池化向量以及噪声作为解 码器的初始态, 同时将观测序列的最后位置点作 为解码器的即时输人, 即可输出预测序列的首个 轨迹点和编码器传递的隐藏态. 在后续计算中, 将 前一刻的预测轨迹点通过空时社交池化网络和隐藏 态混合输人解码器, 直到最终输出全部预测轨迹.

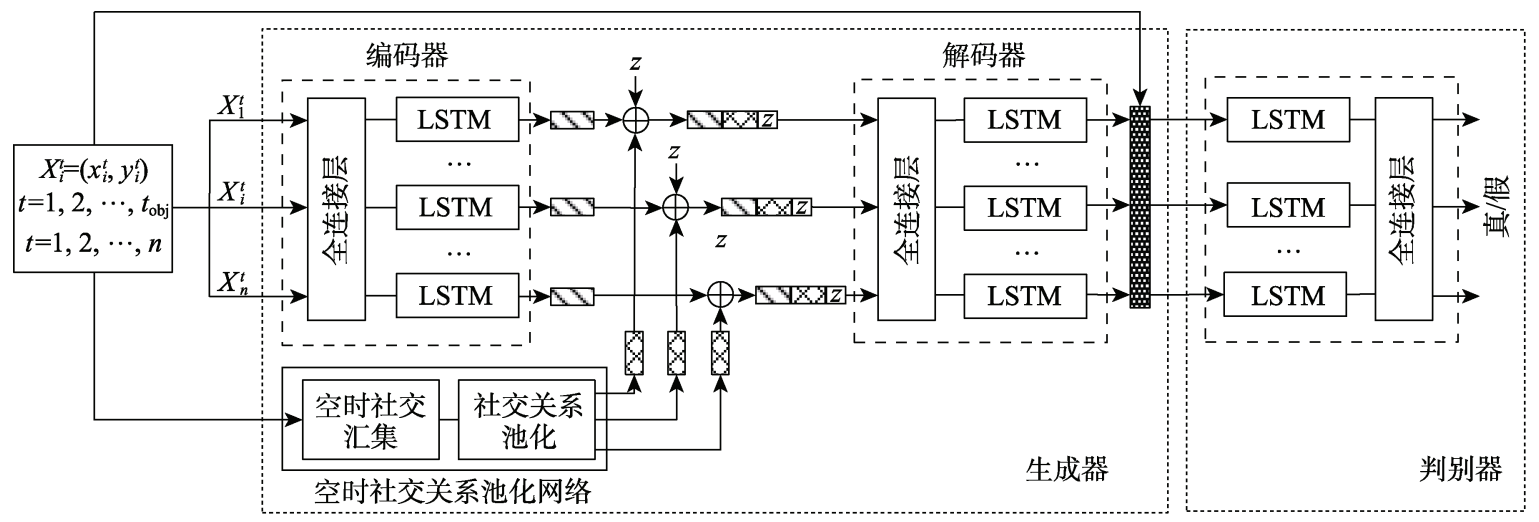

图 2 SOPM 结构示意图 


\section{3 空时社交汇集模块}

行人在空间中的社交本质上也是一组关于图 时间的序列, 行人社交可以表示为以该行人为中 心与所有行人的空间距离.

定义 2. 设第 $i$ 个行人在 $t$ 时刻的轨迹为 $X_{i}^{t}$, 则该行人 $i$ 在 $t$ 时刻的所有社交可以用空间距离表 示为

$$
S_{i}^{t}=\left\{X_{i}^{t}-X_{j}^{t}\right\}, j=1,2, \cdots, n
$$

模型在解码器预测轨迹阶段, 需要将编码器 获得隐藏态和社交池化网络得到的社交池化向量 混合编码作为编码器的初始态, 要求隐藏态和社 交池化向量的维度一致. 因此本文使用一种空时 社交汇集机制，利用 RNN 学习连续时间内的社交 信息，可以获得一个如图 3 所示包含长时社交信息 的社交汇集向量.

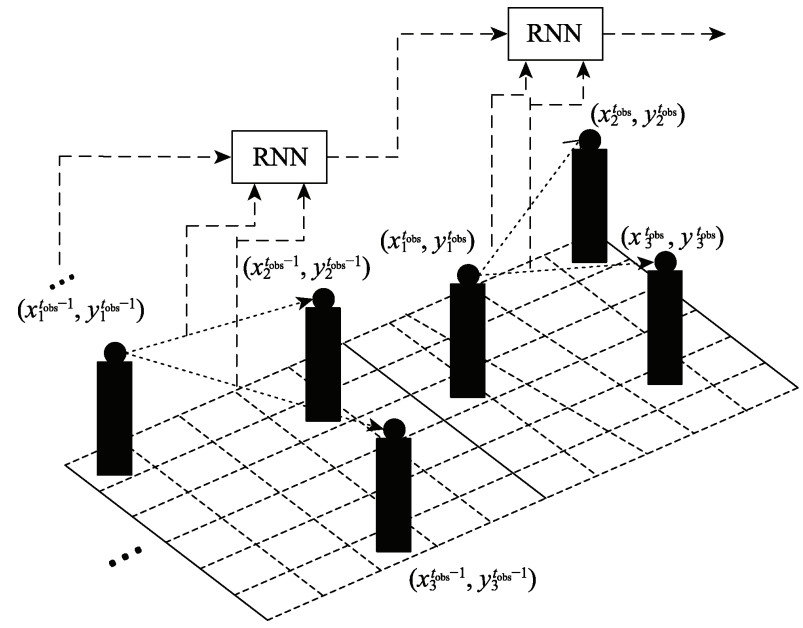

图 3 社交向量汇集示意图

时空社交汇集机制在编码器运行阶段, 首先 通过空间距离计算得到行人观测序列的社交，将 同一时刻的 $n$ 组值依次输人 LSTM，每次计算中利 用上一刻的映射向量来初始化 LSTM, 实现对行人 的社交化长时分析.

设 $\boldsymbol{h}_{i}^{p}(t-1)$ 为上一时刻的社交化隐藏态输出, $\boldsymbol{W}_{p}$ 是社交 LSTM 权重, 则 $t$ 时刻第 $i$ 个行人的社交 汇集向量为

$$
\boldsymbol{h}_{i}^{p}(t)=\operatorname{LSTM}\left(h_{i}^{p}(t-1), S_{i}^{t-1}, \boldsymbol{W}_{p}\right)
$$

其中, $t=1,2, \cdots, t_{\mathrm{obs}}$, 获得的社交汇集向量为 $\boldsymbol{h}_{i}^{p}\left(t_{\mathrm{obs}}\right)$.

在解码器阶段, 编码器隐藏态与该时刻社交 池化向量混合初始化解码器, 将上一时刻轨迹点 作为解码器即时输人, 输出的轨迹点即为预测值.
与编码器时阶段空时社交汇集不同, 解码器阶段利 用预测的轨迹点作为输人, 由前一刻获得社交汇集 向量值和编码器阶段获得汇集向量共同作为隐藏态 传递. 则 $t$ 时刻第 $i$ 个行人的社交汇集向量为

$$
\boldsymbol{h}_{i}^{p}(t)=\operatorname{LSTM}\left(\left(\boldsymbol{h}_{i}^{p}\left(t_{\mathrm{obs}}\right), \boldsymbol{h}_{i}^{p}(t-1)\right), S_{i}^{t-1}, \boldsymbol{W}_{p}\right)
$$

其中, $t=t_{\mathrm{obs}}+1, \cdots, t_{\mathrm{obs}}+t_{\text {pred }}$.

\section{4 社交关系池化模块}

时空社交汇集获得了行人的社交化汇集向量, 是行人关联空间中所有行人的时序信息, 但编码 器每个独立 RNN 隐藏态只包含行人个体的时序信 息，使得社交汇集向量的信息量是轨迹隐藏态信 息量的数倍，必须采取有效的方法结合两者的信 息. 关系池化的作用是将社交汇集向量正确池化 获得有用的社交池化向量. 考虑到空间中行人的 社交关系简化为“吸引”和“排斥”最合适，将社交化 汇集向量与轨迹的隐藏态混合, 池化为“引力”和 “质力”社交向量，混合这 2 种社交池化向量和高斯 噪声即为解码器的输人.

定义 3. 在编码器学习观测序列时, 设 $t-1$ 时 刻第 $i$ 个行人在编码器中的隐藏态为 $\boldsymbol{h}_{i}^{e}(t-1)$, 则 编码器输出的隐藏态为

$$
\begin{aligned}
\boldsymbol{e}_{i}(t) & =\gamma\left(x_{i}(t), y_{i}(t), \boldsymbol{W}_{e e}\right) \\
\boldsymbol{h}_{i}^{e}(t) & =\operatorname{LSTM}\left(\boldsymbol{h}_{i}^{e}(t-1), \boldsymbol{e}_{i}(t), \boldsymbol{W}_{e n}\right)
\end{aligned}
$$

其中， $\left(x_{i}(t), y_{i}(t)\right)$ 为当前位置行人轨迹点; $\gamma$ 为全 连接层组成的线性变化; $\boldsymbol{W}_{e e}$ 是线性变换的权重, $\boldsymbol{W}_{e n}$ 为编码器权重.

社交汇集向量和编码器隐藏态混合后池化, 则引力社交池化向量为

$$
\max \boldsymbol{h}_{i}^{p e}(t)=\max \left\{\left(\phi\left(\boldsymbol{h}_{i}^{e}(t)\right), \boldsymbol{h}_{i}^{p}(t)\right)\right\}
$$

其中, $\phi$ 为线性变化函数; $\boldsymbol{h}_{i}^{p}(t)$ 为由时空社交汇 集机制输出的社交汇集向量; $\boldsymbol{h}_{i}^{e}(t)$ 为编码器学习 观测序列轨迹点输出的隐藏态. 则斥力社交池化 向量为

$$
\min \boldsymbol{h}_{i}^{p e}(t)=\min \left\{\left(\phi\left(\boldsymbol{h}_{i}^{e}(t)\right), \boldsymbol{h}_{i}^{p}(t)\right)\right\}
$$

利用获得的社交池化向量和噪声 $z$ 共同作为 解码器隐藏态, 则解码器输出为

$$
\begin{gathered}
\boldsymbol{e}_{i}(t)=\delta\left(x_{i}(t), y_{i}(t), \boldsymbol{W}_{e d}\right) \\
\hat{Y}_{i}(t), \boldsymbol{h}_{i}^{d}(t)=\operatorname{LSTM}\left(\boldsymbol{e}_{i}(t),\left(\max \boldsymbol{h}_{i}^{p e}(t-1) \oplus\right.\right. \\
\left.\left.\min \boldsymbol{h}_{i}^{p e}(t-1), z\right), \boldsymbol{W}_{\mathrm{dn}}\right)
\end{gathered}
$$

其中, $\left(x_{i}(t), y_{i}(t)\right)$ 为行人的轨迹点; $\delta$ 为全连接 层组成的线性变化函数; $\boldsymbol{W}_{e d}$ 是线性变换的权重, 
$\boldsymbol{W}_{d n}$ 为解码器权重; $\oplus$ 表示维度拼接.

\subsection{SOPM 原理特点}

(1) 空时社交汇集机制旨在将行人社交的空 间距离变换到与 RNN 编码器隐藏态维度相同的 域，本质上属于一种映射方式. SGAN 社交汇集方 法的映射如图 4a 所示, 通过全连接网络将空间距 离映射到隐藏态域. 本文社交汇集方法的映射如 图 4b 所示, 通过 RNN 将连续时间内的空间距离最 终映射到隐藏态域. 相比于 SGAN 只从空间映射 的方法，本文方法从时间空间上映射得到社交汇 集向量，能利用更多的轨迹信息，对行人社交做出 更有效的分析.

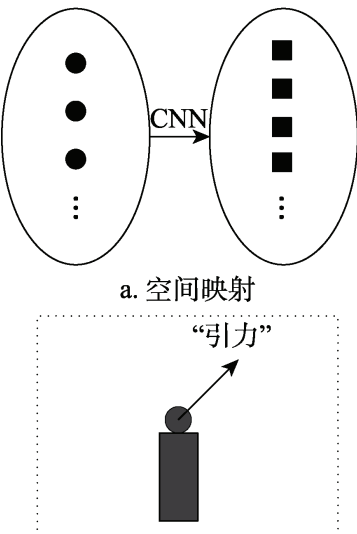

c. SGAN社交关系

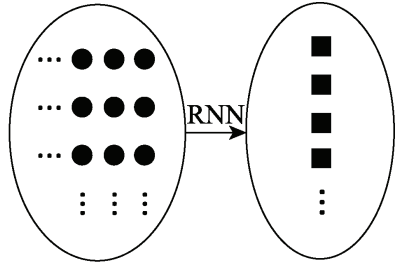

b. 空时映射 “引力”

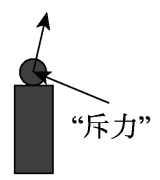

d. SPOM社交关系
图 42 种社交汇集原理对比示意图

（2）社交池化是为了由社交汇集向量获得有 效的社交池化向量, 可以看做从行人所有的社交 关系中简化出关键的关系. 如图 4c 所示, SGAN 的 池化方法是寻找其中相关度最大的一对向量，可 以认为是只利用了引力关系. 如图 4d 所示, 本文 方法采用相关度最大和最小的 2 对向量，同时利用 引力和斥力关系，使得模型可以对“避让”等行人 轨迹做出很好的预测。

\section{3 模型训练细则}

模型在训练过程中, 生成器 $G$ 负责学习观测 序列在特征空间里的分布, 来生成一个与真实轨 迹尽可能接近的生成轨迹, 直到判别器 $D$ 难以对 生成样本和真实样本做出区分, 则认为生成器训 练完成.

设生成器输人为 $x$, 随机噪声为 $z$, 则模型的 训练过程为

$$
\begin{gathered}
\min _{G} \max V(G, D)=E_{D} \sim P \operatorname{data}(x) \\
E_{z \sim P z(z)}(\log D(x))+ \\
(\log (1-D(G(z))))
\end{gathered}
$$

为了保证模型生成样本的多样性, 采用 $L_{2}$ 多 样损失来保证生成最佳轨迹, 即由生成器同时生 成同一行人的多条轨迹，找到其中损失最小的轨 迹, $k$ 为采用的多样损失, 则计算公式为

$$
L=\min _{k}\left\|Y_{i}-\hat{Y}_{i}\right\|^{2}
$$

算法的训练步骤如下:

Step1. 准备训练数据, 并对数据预处理, 将包含行 人轨迹的连续序列分成观测序列和真实序列, 共同作为 训练样本，其中真实序列的标签全部为 1 .

Step2. 设定训练样本的批次为 batch, 所有的样本 为一个 epoch, 将 epoch 分为 batch 个训练序列, 每个 batch 序列包含同一空间中的多行人运动轨迹.

Step3. 计算一个批次训练样本中的行人观测数据 的坐标差值, 输人生成器的 RNN 编码器, 得到观测序 列的隐藏态.

Step4. 将同一批次中行人的观测序列输人社会关 系池化, 得到行人社交化程度的池化向量, 结合编码器 的隐藏态, 共同作为解码器的初始态.

Step5. 将观测序列的最后一个行人坐标差值输人 解码器, 依次输出预测序列, 设置预测序列的标签为 0 .

Step6. 将真实序列和观测序列组成的真实样本和 预测序列和观测序列组成的生成样本作为判别器的输 人, 输出判定结果. 根据标签值计算损失, 保证生成器 的权重不变, 更新判别器的权重梯度.

Step7. 执行与 Step3 Step6 相同的操作 1 次, 提高 判别器分辨生成样本和真实样本的能力, 以便后续尽快 地获得合适的生成器.

Step8. 执行与 Step3 Step4 相同的操作 1 次, 将观 测序列的最后一个行人坐标差值输人解码器, 依次输出 预测序列, 设置预测序列的标签为 1. 将真实样本和生 成样本作为判别器的输人, 根据标签值计算损失, 保证 判别器的权重梯度不变, 更新生成器的权重.

Step9. 每隔固定批次, 通过数据集中的验证数据 对模型的精度进行检测, 并将其中精度最佳的模型结构 和权重保存. 直到设定的 epoch 训练完成, 保存验证集 表现最好的模型参数.

\section{4 实验结果与分析}

本文的实验环境为 Ubuntu16.04 系统, NVIDIA GTX 1080Ti GPU, Intel Xeon CPU E5-2683, 同时 采用 Python v3 版本的 Pytorch 0.4.1 框架，运行环 境为 CUDA 9.0. 为了验证本文提出的模型是否具 有更好的性能, 使用了 2 个公开数据集 $\mathrm{ETH}^{[14]}$ 和 $\mathrm{UCY}^{[15]}$. 这 2 个数据集都是在现实世界采集获得 的多行人交互的轨迹序列, 被广泛用于各种轨迹 预测模型的性能比较, 其中 ETH 包含 ETH 和 Hotel 
2 个场景, UCY 包含 UNIV, ZARA1 和 ZARA2 3 个 场景.

\section{1 预测精度}

为了对提出模型的预测性能进行很好的评估, 本文使用了平均偏移精度误差 (average deviation accuracy error, ADE)和最终偏移精度误差 (final deviation accuracy error, FDE) 来衡量 ${ }^{[16]}$, 其中 ADE 用于比较整个预测序列与真实轨迹, FDE 关注预测 序列的最后轨迹点与真实轨迹点的相近程度. 若有 $N$ 条轨迹, $m$ 为轨迹的长度, 则计算公式分别为

$$
\begin{gathered}
\mathrm{ADE}=\frac{1}{N} \sum_{i=1}^{N} \frac{1}{t^{m}}\left(\left(x_{i}(t)-\hat{x}_{i}(t)\right)+\left(y_{i}(t)-\hat{y}_{i}(t)\right)\right)^{\frac{1}{2}} \\
\mathrm{FDE}=\frac{1}{N} \sum_{i=1}^{N}\left(\left(x_{i}\left(t_{\mathrm{pred}}\right)-\hat{x}_{i}\left(t_{\mathrm{pred}}\right)\right)+\right. \\
\left.\left(y_{i}\left(t_{\mathrm{pred}}\right)-\hat{y}_{i}\left(t_{\mathrm{pred}}\right)\right)\right)^{\frac{1}{2}}
\end{gathered}
$$

SOPM-s 表示单独采用空时社交汇集机制的模 型, SOPM-p 表示单独采用社交关系池化模块的模 型, SOPM-s-p(简称 SOPM) 表示同时采用空时社交 汇集机制和社交关系池化模块的模型. 为了观察
本文模型与其他模型相比性能是否有所提升, 本文使用 Linear, S-LSTM ${ }^{[6]}$ 和 SGAN ${ }^{[7]}$ 共同测试, 作为实验对比对象. 该实验使用步长为 $8 \mathrm{~m}$ 的 $\mathrm{ADE}$ 和 FDE 作为比较指标, 具体的实验结果如 表 1 所示.

从各个数据集的表现来看, 在 $\mathrm{ETH}$ 中, 无论 是 $\mathrm{ADE}$ 还是 FDE, SOPM 都表现最为出色. 在 HOTEL 中, 由于行人轨迹表现较为平缓均匀, 因 此 Linear 表现最好, 其中 SOPM-s 的 ADE 也较为 接近 Linear 模型, 可见使用空时社交汇集机制能 较好地控制 SGAN 对行人顺滑轨迹拟合不好的问 题. 在 UNIV 这种行人较多的轨迹场景中, SOPM-s 由于能对观测序列的社交关系进行记忆学习, 所 以 ADE 强于 SOPM-p 和 SOPM. 在 ZARA1 中, SOPM-p 的 ADE 最高, 并且改进后的 3 个模型性 能都高于 SGAN. 在 ZARA2 中, 只采用社交关系 池化的 SOPM-s 的 ADE 最好. 从所有数据集综合 的平均值来看, SOPM 都是表现最为出色, 相较于 $\mathrm{SGAN}$, 平均 $\mathrm{ADE}$ 提升 $20 \%$, 平均 $\mathrm{FDE}$ 提升

\begin{tabular}{|c|c|c|c|c|c|c|c|c|c|c|c|c|}
\hline \multirow{2}{*}{ 模型 } & \multicolumn{5}{|c|}{$\mathrm{ADE}$} & \multicolumn{7}{|c|}{ FDE } \\
\hline & ETH & HOTEL & UNIV & ZARA1 & ZARA2 & 平均 & ETH & HOTEL & UNIV & ZARA1 & ZARA2 & 平均 \\
\hline Linear & 0.84 & 0.35 & 0.56 & 0.41 & 0.53 & 0.54 & 1.60 & 0.60 & 1.01 & 0.56 & 0.95 & 0.98 \\
\hline S-LSTM & 0.73 & 0.49 & 0.41 & 0.27 & 0.33 & 0.43 & 1.48 & 1.01 & 0.84 & 0.71 & 0.79 & 0.91 \\
\hline SGAN & 0.69 & 0.49 & 0.40 & 0.22 & 0.22 & 0.45 & 1.47 & 1.05 & 0.75 & 0.45 & 0.58 & 0.86 \\
\hline SOPM-s & 0.67 & 0.37 & 0.32 & 0.23 & 0.21 & 0.36 & 1.33 & 0.72 & 0.70 & 0.49 & 0.46 & 0.74 \\
\hline SOPM-p & 0.64 & 0.43 & 0.33 & 0.22 & 0.20 & 0.37 & 1.28 & 0.87 & 0.70 & 0.46 & 0.48 & 0.75 \\
\hline SOPM & 0.61 & 0.40 & 0.34 & 0.23 & 0.21 & 0.36 & 1.27 & 0.81 & 0.68 & 0.49 & 0.45 & 0.74 \\
\hline
\end{tabular}
$13.9 \%$.

表 1 不同数据集上 ADE 和 FDE 实验结果

注. 粗体的数值为具体场景下最高精度值.

从 $\mathrm{ADE}$ 来看, SOPM-s 对存在较多长时社交的 HOTEL 预测精度较好, SOPM-p 在行人关系复杂的 ZARA1 和 ZARA2 表现最好, SOPM 虽然在部分数 据集上弱于 SOPM-s 和 SOPM-p, 但平均值在 3 个 模型表现最好. 从 FDE 来看, SOPM 始终强于 SOPM-s 和 SOPM-p. 这意味着 2 种方法组合的模 型虽然在单独数据集上表现不一定好, 但整体上 提升了模型的预测性能.

\section{2 预测效率}

本文的实验结果除了各模型的预测精度比较, 还包括上文提到的各个模型的预测效率比较. 为 了更全面地衡量模型的预测效率，本文采用了平 均训练时间和平均预测时间 2 个指标, 其中训练时 间指的是训练单组所有行人轨迹所需的平均时间;
预测时间是对单组行人序列预测所花费的平均时 间，实验结果如表 2 所示.

表 2 预测效率实验结果

\begin{tabular}{lcc}
\hline \multicolumn{1}{c}{ 模型 } & 平均训练时间 $/ \mathrm{min}$ & 平均预测时间 $/ \mathrm{ms}$ \\
\hline Linear & 14.5 & 2.9 \\
S-LSTM & 22.8 & 5.2 \\
SGAN & 61.4 & 31.5 \\
SOPM-s & 73.2 & 41.6 \\
SOPM-p & 62.3 & 32.4 \\
SOPM & 74.6 & 42.7 \\
\hline
\end{tabular}

由表 2 可知, Linear 模型由于采用了线性预测, 模型简单, 2 个指标都表现最好. S-LSTM 模型因为 使用了社会池化网格方法, 较 Linear 实时性有所 
下降. SGAN 由于使用了生成对抗网络的方法, 算 法复杂度更高，使得该模型可在提高精度的同时 避免预测效率的降低. SOPM-s 相比于 SGAN, 在 社交池化中采用了 RNN 的向量汇集机制，而不是 简单的全连接网络, 因为 RNN 计算所需花销要大 于全连接网络, 使得该模型较 SGAN 略有下降. SOPM-p 虽然采用了社交关系池化，但整体计算量 与 SGAN 一致, 预测效率整体不变. SOPM 同时采 用了空时社交汇集机制和关系池化，使得该模型
相较于其他模型, 所需的平均训练时间和平均预 测时间都有所增加, 但相比于 SGAN, 在较小地 牺牲预测效率的同时, 极大地提升了数据集预测 精度.

\section{3 场景分析}

本节使用数据集与上述实验一致，旨在通过 在一些特定场合的行人轨迹分析，对各个模型的 优点和缺陷进行分析, 并对本文模型的实际效果 进行验证, 如图 5 所示.

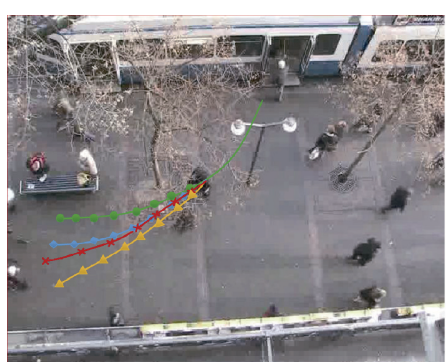

a. 躲避行人轨迹发生偏转的情况

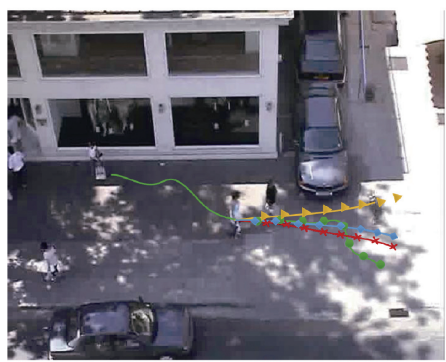

d. 行人出现复杂轨迹的情况

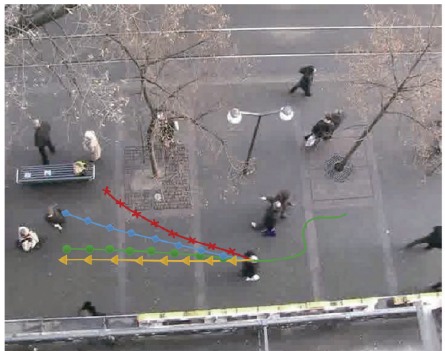

b. 快速经过一对行人的情况

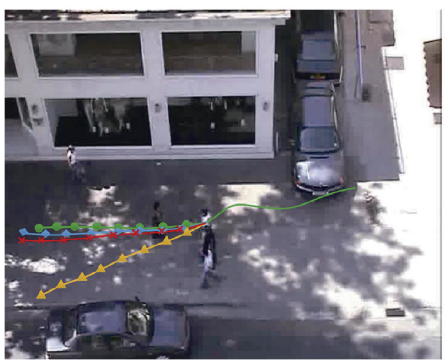

e. 朋友相伴而行的情况

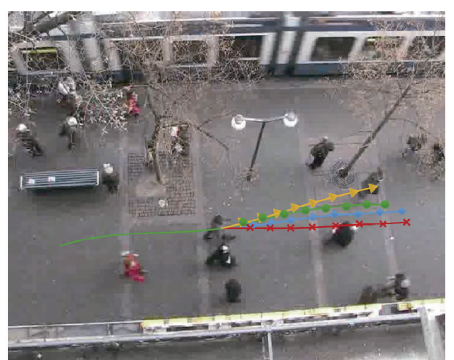

c. 与另一行人迎面相遇的情况

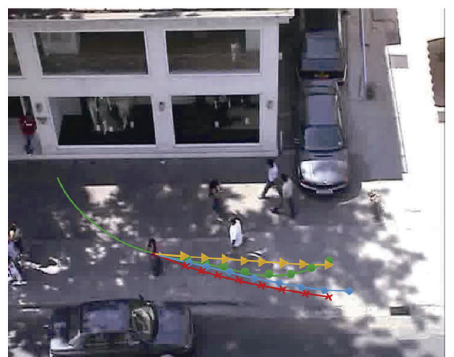

f. 行进中途突然转弯的情况

— 真实轨迹;

S-LSTM;

S-GAN;

SOPM

图 5 场景分析示意图

图 5a 所示为一对朋友下车后因为躲避行人而 轨迹发生偏转的情况, 其中 SOPM 和 SGAN 表现 与原有轨迹更为接近, S-LSTM 表现最差. 图 5b 所 示为一个行人快速经过一对行人的轨迹路线，其 中 S-LSTM 表现得最为出色，而 SGAN 由于社交池 化过程中过分关注短时社交，使得该行人轨迹偏 向同向的另一对行人, SOPM 由于使用了空时社交 汇集机制, 较 SGAN 表现较好. 图 5c 所示为一对 朋友与另一行人迎面相遇的情况, 其中 SGAN 表 现最差，而 SOPM 因为使用了社交关系池化表现 更好. 图 5d 所示为行人出现复杂轨迹的情况, 其 中 S-LSTM 对轨迹的预测更接近为光滑路线, 表现 最差, SOPM 和 SGAN 的预测轨迹与真实轨迹大体 一致. 图 5e 所示为一对朋友相伴而行的情况, 其 中 S-LSTM 由于行人社交化考虑不足, 表现不佳,
$\mathrm{SOPM}$ 与 SGAN 相比, 更接近真实行人轨迹. 图 $5 \mathrm{f}$ 所示为行人在行进中途突然转弯的情况, 该情况 下, S-LSTM 总体预测轨迹更为平滑，与真实轨迹 重叠处较多, SOPM 的预测轨迹比 SGAN 的预测更 接近真实轨迹.

\section{5 结 语}

本文通过使用公开的标准行人轨迹数据集与 各种模型做出比对实验, 实验结果表明, 相比 SGAN, SOPM 表现较为出色. 空时社交汇集机制很好地 解决了行人过分关注短时社交的风险，模型在长 时社交时轨迹上的精度有所提高. 使用社交关系 池化有效地解决了行人避让等轨迹突变情况下行 人的社交化影响. 本文模型虽然很好地解决了 
SGAN 存在的问题，但也存在一个现有模型共同 的缺陷, 就是无法很好地将行人的自身轨迹与其 社交化影响结合起来，常导致预测偏离受社交影 响过大，因此，正确地处理社交池化网络与 RNN 编码-解码器的信息融合将是下一步的研究重点.

\section{参考文献(References):}

[1] Large F, Vasquez D, Fraichard T, et al. Avoiding cars and pedestrians using V-obstacles and motion prediction[C] //Proceedings of the IEEE Intelligent Vehicle Symposium. Los Alamitos: IEEE Computer Society Press, 2004: 375-379

[2] Thompson S, Horiuchi T, Kagami S. A probabilistic model of human motion and navigation intent for mobile robot path planning[C] //Proceedings of the International Conference on Autonomous Robots and Agents. Los Alamitos: IEEE Computer Society Press, 2009: 663-668

[3] Wang J M, Fleet D J, Hertzmann A. Gaussian process dynamical models for human motion[J]. IEEE Transactions on Pattern Analysis and Machine Intelligence, 2008, 30(2): 283-298

[4] Helbing D, Molnar P. Social force model for pedestrian dynamics[J]. Physical Review E: Statistical Physics Plasmas Fluids and Related Interdisciplinary Topics, 1995, 51(5): 4282-4286

[5] Chung J, Kastner K, Dinh L, et al. A recurrent latent variable model for sequential data[C] //Proceedings of the 28th International Conference on Neural Information Processing Systems. Cambridge: MIT Press, 2015: 2980-2988

[6] Alahi A, Goel K, Ramanathan V, et al. Social LSTM: human trajectory prediction in crowded spaces[C] //Proceedings of the IEEE Conference on Computer Vision and Pattern Recognition. Los Alamitos: IEEE Computer Society Press, 2016: 961-971

[7] Gupta A, Johnson J , Li F F, et al. Social GAN: socially acceptable trajectories with generative adversarial networks[C] //Proceedings of the IEEE/CVF Conference on Computer Vision and Pattern Recognition. Los Alamitos: IEEE Computer Society Press, 2018: 2255-2264
[8] Yu P, Song K T, Lu J F. Generating adversarial examples with conditional generative adversarial net[C] //Proceedings of the 24th International Conference on Pattern Recognition. Los Alamitos: IEEE Computer Society Press, 2018: 676-681

[9] Katranji M, Sanmarty G, Moalic L, et al. RNN encoder-decoder for the inference of regular human mobility patterns[C] //Proceedings of the International Joint Conference on Neural Networks. Los Alamitos: IEEE Computer Society Press, 2018:1-9

[10] Huang Y F, Bi H K, Li Z X, et al. STGAT: modeling spatial-temporal interactions for human trajectory prediction[C] //Proceedings of the IEEE/CVF International Conference on Computer Vision. Los Alamitos: IEEE Computer Society Press, 2019: 6272-6281

[11] Kosaraju V, Sadeghian A, Martín-Martín R, et al. Social-BiGAT: multimodal trajectory forecasting using bicycle-GAN and graph attention networks[OL]. [2020-02-15]. https://arxiv.org/abs/1907.03395v1

[12] Kitani K M, Ziebart B D, Bagnell J A, et al. Activity forecasting $[\mathrm{C}] / /$ Proceedings of European Conference on Computer Vision. Heidelberg: Springer, 2012: 201-214

[13] Leal-Taixé L , Fenzi M , Kuznetsova A, et al. Learning an image-based motion context for multiple people tracking[C] //Proceedings of the IEEE Conference on Computer Vision and Pattern Recognition. Los Alamitos: IEEE Computer Society Press, 2014: 3542-3549

[14] Pellegrini S, Ess A, van Gool L. Improving data association by joint modeling of pedestrian trajectories and groupings[C] //Proceedings of the 11th European Conference on Computer Vision. Heidelberg: Springer, 2010: 452-465

[15] Lerner A, Chrysanthou Y, Lischinski D. Crowds by example[J]. Computer Graphics Forum, 2007, 26(3): 655-664

[16] Lee N, Choi W, Vernaza P, et al. Desire: distant future prediction in dynamic scenes with interacting agents[C]//Proceedings of the IEEE Conference on Computer Vision and Pattern Recognition. Los Alamitos: IEEE Computer Society Press, 2017: 2165-2174 